\title{
Moment-free numerical approximation of highly oscillatory integrals with stationary points
}

\author{
Sheehan Olver *
}

\begin{abstract}
We present a method for the numerical quadrature of highly oscillatory integrals with stationary points. We begin with the derivation of a new asymptotic expansion, which has the property that the accuracy improves as the frequency of oscillations increases. This asymptotic expansion is closely related to the method of stationary phase, but presented in a way that allows the derivation of an alternate approximation method that has the same asymptotic behaviour, but with significantly greater accuracy. This approximation method does not require moments.
\end{abstract}

\section{Introduction}

We are concerned with numerically approximating the highly oscillatory integral

$$
I[f]=\int_{-1}^{1} f(x) \mathrm{e}^{\mathrm{i} \omega g(x)} \mathrm{d} x
$$

where $\omega$ is large,

$$
0=g(0)=g^{\prime}(0)=\cdots=g^{(r-1)}(0), \quad g^{(r)}(0)>0,
$$

and $g^{\prime}(x) \neq 0$ for $0<|x| \leq 1$. Note that the case where $g(0) \neq 0$ can easily be handled by replacing $g$ with $g-g(0)$, and multiplying the integral by $\mathrm{e}^{\mathrm{i} \omega g(0)}$. The condition that $g^{(r)}(0)>0$ implies that $g(x)>0$ for $0<x \leq 1$, and $(-1)^{r} g(x)>0$ for $-1 \leq x<0$. This condition, as well as the continuity of $g$ and $f$ can be relaxed, at the expense of complicating the proofs.

Computing such integrals using traditional techniques, such as Gaussian quadrature, is difficult. Accuracy depends on there being several samples per oscillation, which for large frequency is extremely inefficient. Fixing the number of quadrature points results in an error $\mathcal{O}(1)$ as $\omega \rightarrow \infty$, whilst the integral itself decays like $\mathcal{O}\left(\omega^{-\frac{1}{r}}\right)[\mathbf{9}]$. The method of stationary phase [5] provides only an asymptotic result; for fixed frequency the accuracy of the approximation is limited. It is possible to compute the integrals by moving to the complex plane and integrating along the path of steepest descent $[\mathbf{3}]$, however, this suffers from the requirement that both $f$ and $g$ are analytic, in order to deform the integration path, and requires the knowledge or computation of the path of steepest descent. Filon-type methods allow efficient approximation [4], however, they require the knowledge of moments $I\left[x^{k}\right]$, which in general are unknown.

In this paper we will present a new method such that the accuracy actually improves as the frequency increases, up to any chosen asymptotic order. We begin by constructing a new asymptotic expansion that does not require moments. From this asymptotic expansion we can find a basis for a Filon-type method which can be integrated explicitly in closed form. This allows us to improve the accuracy of the approximation for fixed asymptotic orders.

\section{Asymptotic Expansion}

Asymptotic expansions provide an invaluable tool for high frequency integration. For the integral in question, there exists two existing asymptotic expansions: the Iserles and Nørsett expansion found in [4] and

* Department of Applied Mathematics and Theoretical Physics, Centre for Mathematical Sciences, Wilberforce Rd, Cambridge CB3 0WA, UK, email: S.0lver@damtp.cam.ac.uk 
the well-known method of stationary phase [5]. The former of these requires knowledge of the moments $I[1]$, $\ldots, I\left[x^{r-1}\right]$ but leads us to the more powerful numerical approximation of Filon-type methods and Levin-type methods [6]. Stationary phase does not require moments, yet only provides an asymptotic result, hence for fixed frequency its usefulness as a numerical quadrature scheme is limited.

In this section, we will derive an asymptotic expansion that bridges the gap between the two: it is roughly equivalent to the method of stationary phase, in that it does not require moments, but presented in a way analogous to the Iserles and Nørsett expansion, allowing us to derive a Filon-type method such that moments are not necessary. We first present the Iserles and Nørsett expansion, to motivate the methodology behind the new expansion. For simplicity, we take $r=2$, though the Iserles and Nørsett expansion exists for higher order stationary points as well, requiring the knowledge of additional moments. The standard technique of deriving asymptotic expansions, namely integration by parts, fails due to the introduction of a singularity at the stationary point. But we can make the singularity removable:

$$
\begin{aligned}
I[f] & =I[f-f(0)]+f(0) I[1]=\frac{1}{\mathrm{i} \omega} \int_{a}^{b} \frac{f(x)-f(0)}{g^{\prime}(x)} \frac{\mathrm{d}}{\mathrm{d} x} \mathrm{e}^{\mathrm{i} \omega g(x)} \mathrm{d} x+f(0) I[1] \\
& =\left[\frac{f(1)-f(0)}{g^{\prime}(1)} \mathrm{e}^{\mathrm{i} \omega g(1)}-\frac{f(-1)-f(0)}{g^{\prime}(-1)} \mathrm{e}^{\mathrm{i} \omega g(-1)}\right]-\frac{1}{\mathrm{i} \omega} I\left[\frac{\mathrm{d}}{\mathrm{d} x}\left[\frac{f(x)-f(0)}{g^{\prime}(x)}\right]\right]+f(0) I[1] .
\end{aligned}
$$

Iterating this process results in an asymptotic expansion. However, this suffers from requiring the first moment, as well as higher order moments when additional derivatives of $g$ vanish at the stationary point.

The idea behind the new expansion is to note that we do not necessarily need to subtract a constant, it is only necessary that the function we subtract is nonzero at the stationary point. Hence we can replace the moments $I\left[x^{k}\right]$, which may not be computable in closed form, with $I\left[\psi_{k}\right]$, where $\psi_{k}$ is constructed in such a way that the integral is guaranteed to be computable. In order to do this, we first look at the canonical case of $g(x)=x^{r}$. Suppose there exists a function $F$ such that

$$
\frac{\mathrm{d}}{\mathrm{d} x}\left[F(x) \mathrm{e}^{\mathrm{i} \omega g(x)}\right]=x^{k} \mathrm{e}^{\mathrm{i} \omega g(x)} .
$$

We can expand out the left-hand side to obtain the following equation:

$$
\mathcal{L}[F]=F^{\prime}+\mathrm{i} \omega g^{\prime} F=x^{k} .
$$

Replacing $g^{\prime}$ with $r x^{r-1}$ we obtain the equation $F^{\prime}+r i \omega x^{r-1} F=x^{k}$. A solution to this equation is known:

$$
F(x)=\frac{\omega^{-\frac{1+k}{r}}}{r} \mathrm{e}^{-\mathrm{i} \omega x^{r}+\frac{1+k}{2 r} \mathrm{i} \pi}\left[\Gamma\left(\frac{1+k}{r},-\mathrm{i} \omega x^{r}\right)-\Gamma\left(\frac{1+k}{r}, 0\right)\right],
$$

where $\Gamma$ is the incomplete gamma function $[\mathbf{1}]$. Incomplete gamma functions are well-known, and can be computed efficiently [2]. In fact, modern mathematical program languages, such as Maple, Mathematica and Matlab (via the mfun function) have very efficient built-in numerical implementations. Intuition suggests that if we replace $x^{r}$ with $g(x)$, then $\mathcal{L}[F]$ will give us the $\psi_{k}$ we were looking for, hopefully independent of $\omega$. The following lemma shows that our intuition is indeed correct:

Lemma 2.1 Let

$$
\phi_{r, k}(x)=D_{r, k}(\operatorname{sgn} x) \frac{\omega^{-\frac{k+1}{r}}}{r} \mathrm{e}^{-\mathrm{i} \omega g(x)+\frac{1+k}{2 r} \mathrm{i} \pi}\left[\Gamma\left(\frac{1+k}{r},-\mathrm{i} \omega g(x)\right)-\Gamma\left(\frac{1+k}{r}, 0\right)\right]
$$

where

$$
D_{r, k}(\operatorname{sgn} x)= \begin{cases}(-1)^{k} & \operatorname{sgn} x<0 \text { and } r \text { even } \\ (-1)^{k} \mathrm{e}^{-\frac{1+k}{r} \mathrm{i} \pi} & \operatorname{sgn} x<0 \text { and } r \text { odd } \\ -1 & \text { otherwise }\end{cases}
$$

Then $\phi_{r, k} \in C^{\infty}[-1,1]$ and, for $\mathcal{L}[F]=F^{\prime}+\mathrm{i} \omega g^{\prime} F$,

$$
\mathcal{L}\left[\phi_{r, k}\right](x)=\operatorname{sgn}(x)^{r+k+1} \frac{|g(x)|^{\frac{k+1}{r}-1} g^{\prime}(x)}{r} .
$$


Furthermore, $\mathcal{L}\left[\phi_{r, k}\right] \in C^{\infty}[-1,1]$. Finally,

$$
I\left[\mathcal{L}\left[\phi_{r, k}\right]\right]=\phi_{r, k}(1) \mathrm{e}^{\mathrm{i} \omega g(1)}-\phi_{r, k}(-1) \mathrm{e}^{\mathrm{i} \omega g(-1)} .
$$

Proof:

The form of $\mathcal{L}\left[\phi_{r, k}\right]$ away from the stationary point follows immediately from the equation for the derivative of the incomplete Gamma function [1]. The continuity of $\mathcal{L}\left[\phi_{r, k}\right]$ follows from the fact that

$$
\frac{\mathrm{d}}{\mathrm{d} x}\left[\operatorname{sgn}(x)^{k+1} \frac{1}{1+k}|g(x)|^{\frac{1+k}{r}}\right]=\mathcal{L}\left[\phi_{r, k}\right](x) \quad x \neq 0,
$$

and

$$
\operatorname{sgn}(x)^{k+1}|g(x)|^{\frac{1+k}{r}}=\operatorname{sgn}(x)^{k+1}\left|\frac{g^{r}(0)}{r !} x^{r}+\mathcal{O}\left(x^{r+1}\right)\right|^{\frac{1+k}{r}}=\left(\frac{g^{r}(0)}{r !}\right)^{\frac{k+1}{r}} x^{k+1}(1+\mathcal{O}(x))^{\frac{k+1}{r}}
$$

is $C^{\infty}[-1,1]$. Combining this with the fact that $\phi_{r, k}$ is continuous ensures that $\phi_{r, k} \in C^{\infty}$. The final integral thus follows from the fundamental theorem of calculus.

Q.E.D.

Remark: The use of sgn and the case statement in the preceding lemma are merely to choose the branch cut so that $\left(x^{r}\right)^{1 / r}=x$ for both positive and negative $x$.

We can also prove that $\left\{\mathcal{L}\left[\phi_{r, k}\right]\right\}$ is a Chebyshev set $[\mathbf{8}]$, hence can interpolate at any given sequence of sample points.

Lemma 2.2 The basis $\left\{\mathcal{L}\left[\phi_{r, k}\right]\right\}$ is a Chebyshev set.

Proof: Let $u=\operatorname{sgn}(x)|g(x)|^{1 / r}$, where $u$ ranges monotonically from $-|g(-1)|^{1 / r}$ to $|g(1)|^{1 / r}$. Let $g_{+}^{-1}(u)$ equal $x \geq 0$ such that $g(x)=u$, and $g_{-}^{-1}(u)$ similarly. When $r$ is odd then $g_{ \pm}^{-1}=g^{-1}$. Note that $\operatorname{sgn} x=\operatorname{sgn} u$, hence $x=g_{\operatorname{sgn} u}^{-1}\left(u^{r}\right)$. Thus we obtain

$$
\sum c_{k} \mathcal{L}\left[\phi_{r, k}\right](x)=\operatorname{sgn}(x)^{r+1} \frac{g^{\prime}(x)|g(x)|^{\frac{1}{r}-1}}{r} \sum c_{k} \operatorname{sgn}(x)^{k}|g(x)|^{\frac{k}{r}}=\frac{g^{\prime}(x) u^{1-r}}{r} \sum c_{k} u^{k} .
$$

It follows that interpolating $f$ by $\mathcal{L}\left[\phi_{r, k}\right]$ is equivalent to interpolating

$$
\frac{r u^{r-1} f(x)}{g^{\prime}(x)}
$$

by the polynomial $\sum c_{k} u^{k}$. This function is clearly well-defined for $u \neq 0$, hence we must show that it is also well-defined for $u=x=0$. But this follows since

$$
\begin{aligned}
\frac{u^{r-1}}{g^{\prime}(x)} & =\frac{\operatorname{sgn}(x)^{r+1}|g(x)|^{1-1 / r}}{g^{\prime}(x)}=\frac{\operatorname{sgn}(x) g(x)}{|g(x)|^{1 / r} g^{\prime}(x)} \\
& =\frac{\left(g_{r} x^{r}+\mathcal{O}\left(x^{r+1}\right)\right)}{\left.x^{r}\left(g_{r}+\mathcal{O}(x)\right)^{1 / r}\right)\left(r g_{r}+\mathcal{O}\left(x^{r}\right)\right)}=\frac{\left(g_{r}+\mathcal{O}\left(x^{r+1}\right)\right)}{\left.\left(g_{r}+\mathcal{O}(x)\right)^{1 / r}\right)\left(r g_{r}+\mathcal{O}\left(x^{r}\right)\right)} .
\end{aligned}
$$
set.

The limit of this as $x$ goes to zero, hence also as $u$ goes to zero, is $\frac{1}{r g_{r}}$. Thus $\mathcal{L}\left[\phi_{r, k}\right]$ is a Chebyshev

Using $\mathcal{L}\left[\phi_{r, k}\right]$ in place of $x^{k}$, we can derive an alternative to the asymptotic expansion in [4], which does not depend on any moments: 
Theorem 2.3 Define $\mu[f]=\sum_{k=0}^{r-2} c_{k} \phi_{r, k}$ so that

$$
\mathcal{L}[\mu[f]](0)=f(0), \ldots, \mathcal{L}[\mu[f]]^{(r-2)}(0)=f^{(r-2)}(0) .
$$

Furthermore, let

$$
\sigma_{0}(x)=f(x), \quad \sigma_{k+1}(x)=\frac{\mathrm{d}}{\mathrm{d} x} \frac{\sigma_{k}(x)-\mathcal{L}\left[\mu\left[\sigma_{k}\right]\right](x)}{g^{\prime}(x)}
$$

Then

$$
\begin{aligned}
& I[f] \sim \sum_{k=0}^{\infty} \frac{1}{(-\mathrm{i} \omega)^{k}}\left\{\mu\left[\sigma_{k}\right](1) \mathrm{e}^{\mathrm{i} \omega g(1)}-\mu\left[\sigma_{k}\right](-1) \mathrm{e}^{\mathrm{i} \omega g(-1)}\right\} \\
& \quad-\sum_{k=0}^{\infty} \frac{1}{(-\mathrm{i} \omega)^{k+1}}\left\{\frac{\sigma_{k}(1)-\mathcal{L}\left[\mu\left[\sigma_{k}\right]\right](1)}{g^{\prime}(1)} \mathrm{e}^{\mathrm{i} \omega g(1)}-\frac{\sigma_{k}(-1)-\mathcal{L}\left[\mu\left[\sigma_{k}\right]\right](-1)}{g^{\prime}(-1)} \mathrm{e}^{\mathrm{i} \omega g(-1)}\right\} .
\end{aligned}
$$

Proof: The proof is based on the proof of Theorem 3.2 in [4]. Note that the existence of such a $\mu$ follows from Lemma 2.2. We find that $\sigma_{k} \in C^{\infty}[0,1]$, since

$$
\frac{\sigma_{k}(x)-\mathcal{L}\left[\mu\left[\sigma_{k}\right]\right](x)}{g^{\prime}(x)}=\frac{\mathcal{O}\left(x^{r-1}\right)}{\frac{g^{r}(0)}{(r-1) !} x^{r-1}+\mathcal{O}\left(x^{r}\right)}=\frac{\mathcal{O}(1)}{\frac{g^{r}(0)}{(r-1) !}+\mathcal{O}(x)}
$$

is $C^{\infty}[0,1]$. Then

$$
\begin{aligned}
I\left[\sigma_{k}\right]= & I\left[\sigma_{k}-\mathcal{L}\left[\mu\left[\sigma_{k}\right]\right]\right]+I\left[\mathcal{L}\left[\mu\left[\sigma_{k}\right]\right]\right] \\
= & \frac{1}{\mathrm{i} \omega} \int_{-1}^{1} \frac{\sigma_{k}-\mathcal{L}\left[\mu\left[\sigma_{k}\right]\right]}{g^{\prime}} \frac{\mathrm{d}}{\mathrm{d} x} \mathrm{e}^{\mathrm{i} \omega g} \mathrm{~d} x+\left\{\mu\left[\sigma_{k}\right](1) \mathrm{e}^{\mathrm{i} \omega g(1)}-\mu\left[\sigma_{k}\right](-1) \mathrm{e}^{\mathrm{i} \omega g(-1)}\right\} \\
= & \frac{1}{\mathrm{i} \omega}\left\{\frac{\sigma_{k}(1)-\mathcal{L}\left[\mu\left[\sigma_{k}\right]\right](1)}{g^{\prime}(1)} \mathrm{e}^{\mathrm{i} \omega g(1)}-\frac{\sigma_{k}(-1)-\mathcal{L}\left[\mu\left[\sigma_{k}\right]\right](-1)}{g^{\prime}(-1)} \mathrm{e}^{\mathrm{i} \omega g(-1)}\right\} \\
& +\left\{\mu\left[\sigma_{k}\right](1) \mathrm{e}^{\mathrm{i} \omega g(1)}-\mu\left[\sigma_{k}\right](-1) \mathrm{e}^{\mathrm{i} \omega g(-1)}\right\}-\frac{1}{\mathrm{i} \omega} I\left[\sigma_{k+1}\right] .
\end{aligned}
$$

The theorem follows from induction.

Q.E.D.

The method of stationary phase can be derived as a consequence of Theorem 2.3. Consider the case of $r$ equal to two. Then $\mu[f](x)=\sqrt{\frac{2}{g^{\prime \prime}(0)}} f(0) \phi_{2,0}(x)$, since $\mathcal{L}\left[\phi_{2,0}\right](0)=\sqrt{\frac{g^{\prime \prime}(0)}{2}}$. If we assume that $g(x) \sim x^{2}$ as $x \rightarrow \pm \infty$, then $\int_{ \pm 1}^{ \pm \infty} f \mathrm{e}^{\mathrm{i} \omega g} \mathrm{~d} x=\mathcal{O}\left(\omega^{-1}\right)[\mathbf{5}]$. Thus formally we obtain

$$
\begin{aligned}
& I[f]= \int_{-\infty}^{\infty} f \mathrm{e}^{\mathrm{i} \omega g} \mathrm{~d} x+\mathcal{O}\left(\omega^{-1}\right)=\int_{-\infty}^{\infty}(f-\mathcal{L}[\mu[f]]) \mathrm{e}^{\mathrm{i} \omega g} \mathrm{~d} x+\int_{-\infty}^{\infty} \mathcal{L}[\mu[f]] \mathrm{e}^{\mathrm{i} \omega g} \mathrm{~d} x+\mathcal{O}\left(\omega^{-1}\right) \\
&= \frac{\mathrm{e}^{\mathrm{i} \pi}}{2 \sqrt{\omega}} \sqrt{\frac{2}{g^{\prime \prime}(0)}} f(0)\left\{\left[\lim _{x \rightarrow \infty} \Gamma\left(\frac{1}{2},-\mathrm{i} \omega g(x)\right)-\Gamma\left(\frac{1}{2}, 0\right)\right]\right. \\
&\left.\quad-\left[\lim _{x \rightarrow-\infty} \Gamma\left(\frac{1}{2},-\mathrm{i} \omega g(x)\right)-\Gamma\left(\frac{1}{2}, 0\right)\right]\right\}+\mathcal{O}\left(\omega^{-1}\right) \\
&=\mathrm{e}^{\frac{\mathrm{i} \pi}{4}} \sqrt{\frac{2 \pi}{\omega g^{\prime \prime}(0)}} f(0)+\mathcal{O}\left(\omega^{-1}\right) .
\end{aligned}
$$

We can demonstrate this asymptotic expansion in action. Note that $\mu\left[\sigma_{k}\right]( \pm 1)=\mathcal{O}\left(\omega^{-1 / r}\right)$, thus the partial sum up to $s-1$ of the asymptotic expansion has an asymptotic order $\mathcal{O}\left(\omega^{-s-1 / r}\right)$. Consider the case where $f(x)=\cos x$ with the polynomial oscillator $g(x)=4 x^{2}+x^{3}$. The moments cannot be 

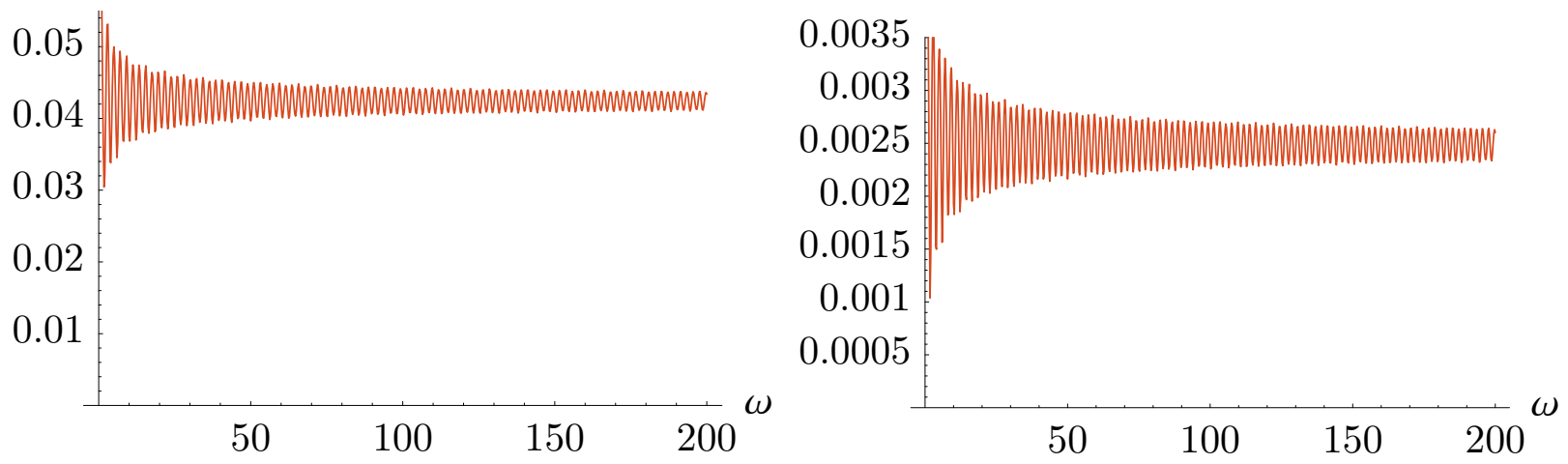

Figure 1: The error scaled by $\omega^{3 / 2}$ of the one-term asymptotic expansion (left-hand figure), versus the error scaled by $\omega^{5 / 2}$ of the two-term asymptotic expansion (right-hand figure), for $\int_{-1}^{1} \cos x \mathrm{e}^{\mathrm{i} \omega\left(4 x^{2}+x^{3}\right)}$.
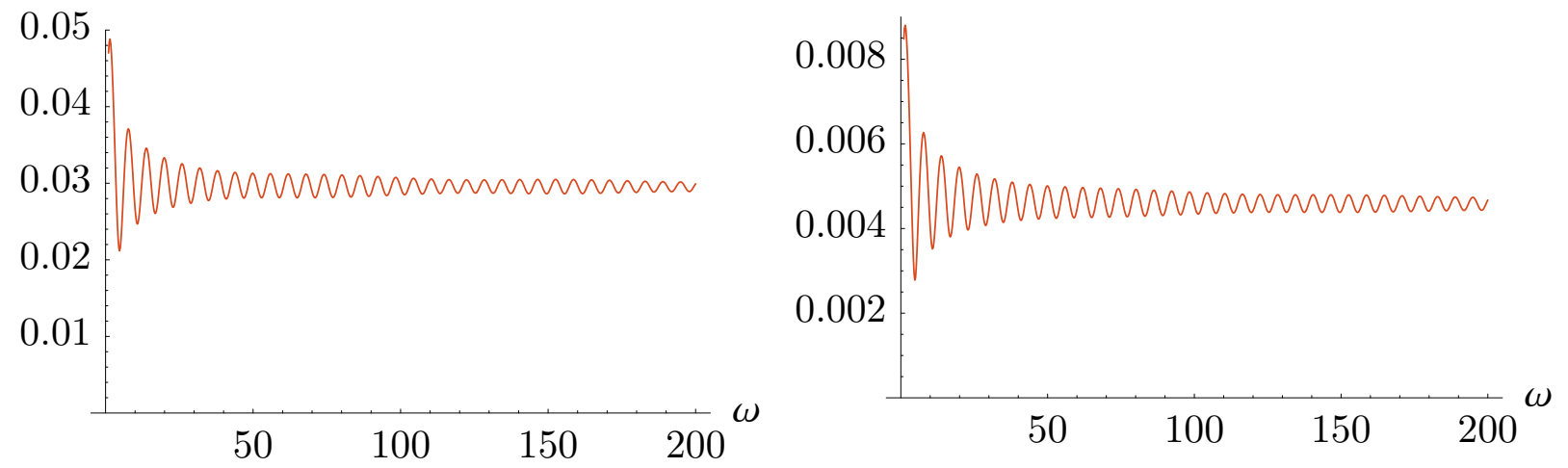

Figure 2: The error scaled by $\omega^{4 / 3}$ of the one-term asymptotic expansion (left-hand figure), versus the error scaled by $\omega^{7 / 3}$ of the two-term asymptotic expansion (right-hand figure), for $\int_{-1}^{1} \frac{1}{x+2} \mathrm{e}^{\mathrm{i} \omega\left(1-\cos x-\frac{1}{2} x^{2}+x^{3}\right)} \mathrm{d} x$.

integrated in closed form, hence the Iserles and Nørsett expansion is not applicable to this integral. On the other hand, Figure 1 demonstrates numerically that Theorem 2.3 does indeed give an asymptotic expansion. For a more complicated example, consider the integral where $f(x)=(x+2)^{-1}$ with the oscillator $g(x)=$ $1-\cos x-\frac{1}{2} x^{2}+x^{3}$. Figure 2 demonstrates that the expansion does indeed work with higher order stationary points, in this case $r$ is three, and with nonpolynomial oscillators.

The following corollary, originally stated in [4], follows from the asymptotic expansion. It is used in the proof of Filon-type methods.

Corollary 2.4 Suppose that

$$
\begin{aligned}
& 0=f(-1)=\cdots=f^{(s-1)}(-1), \\
& 0=f(0)=\cdots=f^{(2 s(r-1)-r)}(0), \\
& 0=f(1)=\cdots=f^{(s-1)}(1) .
\end{aligned}
$$

Then

$$
I[f] \sim \mathcal{O}\left(\omega^{-s-1 / r}\right), \quad \omega \rightarrow \infty
$$

Proof: $\quad$ Note that $\sigma_{k}$ depends on $f$ and its first $k$ derivatives, hence the requirement at the boundary points. We prove the requirement on the number of derivatives at the stationary point by induction. The case where $s=1$ is clear: we need $f$ and its first $r-2$ derivatives to be zero in order for $\mu\left[\sigma_{0}\right]=\mu[f]=0$. The corollary thus follows from L'Hôpital's rule, and the fact that $g^{\prime}$ has a zero of order $r-1$. 


\section{Moment-free Filon-type methods}

The major failing of using an asymptotic expansion as a numerical approximation is that for fixed frequency the expansion in general does not converge. To combat this problem Filon-type methods were developed in [4]. A Filon-type method is constructed by interpolating the function $f$, using the basis functions $\left\{\psi_{k}\right\}$, at a sequence of nodes $\left\{x_{1}, \ldots, x_{\nu}\right\}$ and multiplicities $\left\{m_{1}, \ldots, m_{\nu}\right\}$.

The following theorem is from [4]. It states that we obtain the same asymptotic behaviour as the asymptotic expansion when we use the same number of derivatives at the endpoints of the interval. Unlike an asymptotic expansion, we can add additional interpolation points within the interval to reduce the error for a fixed asymptotic order. In fact, if the interpolant converges uniformly to $f$, then it follows necessarily that the quadrature converges to the exact value of the integral, for fixed $\omega$.

Theorem 3.1 Let $v(x)=\sum_{k=1}^{n} c_{k} \psi_{k}$, where $\psi_{k}$ is independent of $\omega$ and $n=\sum_{k=1}^{\nu} m_{k}$. Assume that $x_{1}=-1, x_{\eta}=0$ and $x_{\nu}=1$. If $c_{k}$ are chosen so that

$$
v\left(x_{k}\right)=f\left(x_{k}\right), \quad \ldots, \quad v^{\left(m_{k}-1\right)}\left(x_{k}\right)=f^{\left(m_{k}-1\right)}\left(x_{k}\right), \quad k=1,2, \ldots, \nu,
$$

then, assuming this system is nonsingular,

$$
I[f]-Q^{F}[f] \sim \mathcal{O}\left(\omega^{-s-1 / r}\right)
$$

when $m_{1}, m_{\nu} \geq s, m_{\eta} \geq(2 s-1)(r-1)$ and

$$
Q^{F}[f]=I[v]=\sum_{k=1}^{n} c_{k} I\left[\psi_{k}\right]
$$

Proof: The theorem follows as a direct consequence of Corollary 2.4:

$$
I[f]-Q^{F}[f]=I[f-v] \sim \mathcal{O}\left(\omega^{-s-1 / r}\right) .
$$

In practice, $\psi_{k}$ is typically defined to be $x^{k}$, i.e., we use standard polynomial interpolation. The reason is two-fold: polynomial interpolation is well understood and guaranteed to interpolate at the given nodes and multiplicities, and the simplicity of the integrand suggests that the moments $I\left[x^{k}\right]$ are likely to be known. However, when the moments are unknown, Filon-type methods with the polynomial basis cannot provide an approximation.

In Lemma 2.1, we determined a basis of functions such that the moments are guaranteed to be known, hence it makes sense to choose $\psi_{k}=\mathcal{L}\left[\phi_{r, k}\right]$ in a Filon-type method. Moreover, it was proved in Lemma 2.2 that $\psi_{k}$ is a Chebyshev set, hence we know that it can interpolate at the given nodes and multiplicities. Thus using this basis we obtain the following theorem:

Theorem 3.2 Let $\psi_{k}=\mathcal{L}\left[\phi_{r, k}\right]$. Then

$$
I[f]-Q^{F}[f] \sim \mathcal{O}\left(\omega^{-s-1 / r}\right)
$$

where

$$
Q^{F}[f]=\sum_{k=1}^{n} c_{k}\left[\phi_{r, k}(1) \mathrm{e}^{\mathrm{i} \omega g(1)}-\phi_{r, k}(-1) \mathrm{e}^{\mathrm{i} \omega g(-1)}\right]
$$



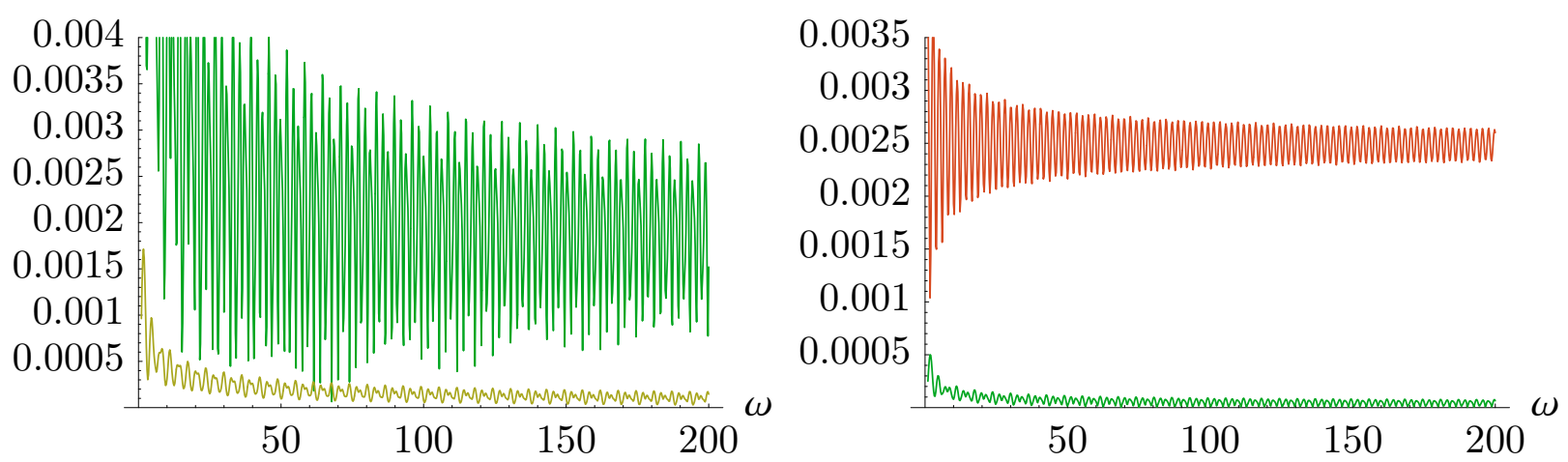

Figure 3: The error scaled by $\omega^{3 / 2}$ of a Filon-type method with endpoints and zero for nodes and multiplicities all one (left figure, top) and a Filon-type method with nodes $\left\{-1,-\frac{1}{2}, 0, \frac{1}{2}, 1\right\}$ and multiplicities all one (left figure, bottom), and the error scaled by $\omega^{5 / 2}$ of the two-term asymptotic expansion (right figure, top) and a Filon-type method with nodes $\{-1,0,1\}$ and multiplicities $\{2,3,2\}$, for $I[f]=\int_{-1}^{1} \cos x \mathrm{e}^{\mathrm{i} \omega\left(4 x^{2}+x^{3}\right)} \mathrm{d} x$.
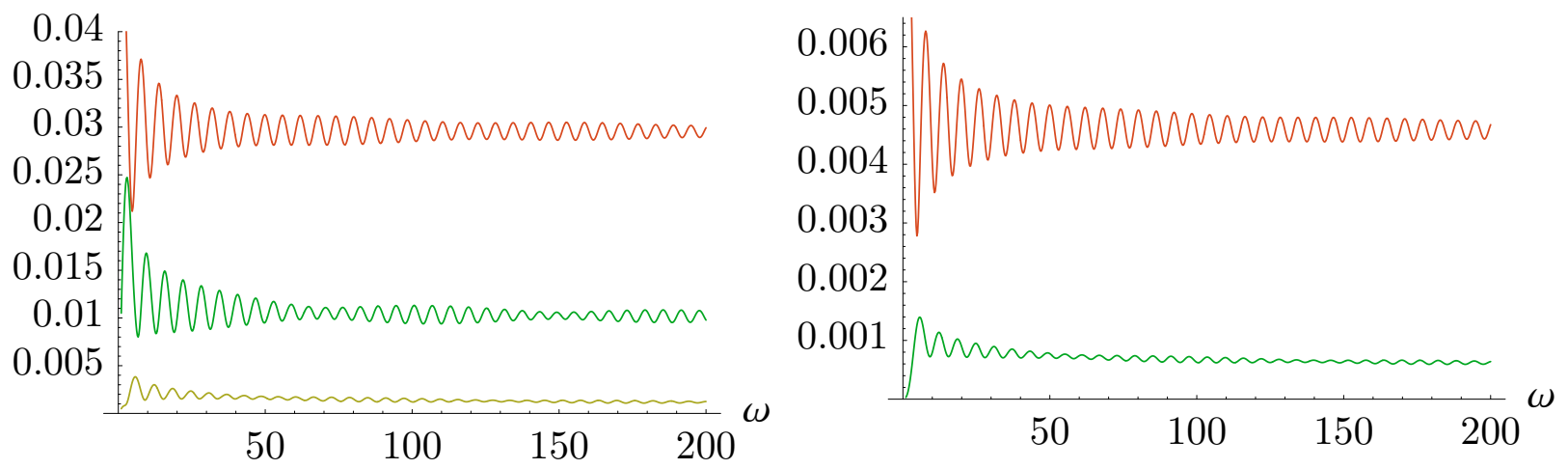

Figure 4: The error scaled by $\omega^{4 / 3}$ of the one-term asymptotic expansion (left figure, top), a Filon-type method with nodes $\{-1,0,1\}$ and multiplicities $\{1,2,1\}$ (left figure, middle) and a Filon-type method with nodes $\left\{-1,-\frac{1}{2}, 0, \frac{1}{2}, 1\right\}$ and multiplicities $\{1,1,2,1,1\}$ (left figure, bottom), and the error scaled by $\omega^{7 / 3}$ of the two-term asymptotic expansion (right figure, top) and a Filon-type method with nodes $\{-1,0,1\}$ and multiplicities $\{2,5,2\}$ (right figure, bottom), for $I[f]=\int_{-1}^{1} \frac{1}{x+2} \mathrm{e}^{\mathrm{i} \omega\left(1-\cos x-\frac{1}{2} x^{2}+x^{3}\right)} \mathrm{d} x$.

Unlike the asymptotic expansion, a Filon-type method allows increasing the accuracy for fixed order. Figure 3 demonstrates this with the same integral as in Figure 1. Note that the errors in the left figure are of the same asymptotic order as the left figure of Figure 1, however the error is significantly less. This is despite the fact that we are using exactly the same information abount $f$ as we are in the asymptotic expansion. Furthermore, this figure demonstrates how adding interpolation points can further reduce the error. The right figure shows how adding sufficient multiplicities to a Filon-type method does indeed increase the asymptotic order, and compares the resulting quadrature with the equivalent asymptotic expansion. We obtain similar results for the integral with a higher-order stationary point found in Figure 2, as seen in Figure 4 .

Remark: We purposely chose oscillators such that $g^{\prime \prime}(x) \neq 0$ for $0<|x|<1$. Without this, $g^{\prime}(x)$ would no longer be monotone and the basis $\mathcal{L}\left[\phi_{r, k}\right]$ would differ greatly in behaviour from the polynomial basis. Though the theorems remain valid, numerical results suggest that $\mathcal{L}\left[\phi_{r, k}\right]$ becomes much less accurate for interpolation, hence a larger amount of sample points would be required. A simple workaround is to choose a sufficiently small neighbourhood around zero such that this condition is satisfied, and use a Momentfree Filon-type method within this neighborhood. We could then approximate the integral outside this 
neighbourhood using a Levin-type method [6]. Like Moment-free Filon-type methods, Levin-type methods do not require moments, however, they cannot be used in the presence of stationary points. They are not affected numerically by $g^{\prime \prime}$ vanishing.

\section{Future work}

It might be possible to generalize these results in the multivariate setting, namely integrating

$$
\int_{\Omega} f(\boldsymbol{x}) \mathrm{e}^{\mathrm{i} \omega g(\boldsymbol{x})} \mathrm{d} V,
$$

where $\Omega$ is piecewise smooth. In [7], the current author derived a quadrature method by using the operator

$$
\mathcal{L}[v]=\nabla \cdot v+\mathrm{i} \omega \nabla g \cdot v
$$

to push the value of the highly oscillatory integral over a domain to a highly oscillatory integral over the boundary, assuming that there are no critical points within the domain: $\nabla g \neq 0$. Initial numerical results suggest that it should be possible to combine these results with the results from this paper in order to derive a numerical approximation in the presence of critical points. We would thus obtain the integral as an integral of incomplete gamma functions over the boundary. There are, however, several major issues. The first problem is that the asymptotics of such integrals is not known in all cases. Indeed, critical points need not be isolated: there can be curves of critical points within the domain. Furthermore, greater care must be taken so that our constructed basis is $C^{\infty}$. Finally, once the integral is pushed to the boundary, there is still the question of how one might integrate the resulting incomplete gamma functions.

\section{References}

[1] Abramowitz, M., and Stegun, I., Handbook of Mathematical Functions, National Bureau of Standards Appl. Math. Series, \#55, U.S. Govt. Printing Office, Washington, D.C., 1970.

[2] Cody, J., An Overview of Software Development, Lecture Notes in Mathematics, 506, Numerical Analysis, Dundee G.A. Watson (ed.), Springer Verlag, Berlin, 1976.

[3] Huybrechs, D., and Vandewalle, S., On the evaluation of highly oscillatory integrals by analytic continuation, SIAM J. Num. Anal., to appear.

[4] Iserles, A., and Nørsett, S.P., Efficient quadrature of highly oscillatory integrals using derivatives, Proceedings Royal Soc. A. 461 (2005), 1383-1399.

[5] Olver, F.W.J., Asymptotics and Special Functions, Academic Press, New York, 1974.

[6] Olver, S., Moment-free numerical integration of highly oscillatory functions, IMA J. Num. Anal. 26 (2006), 213-227.

[7] Olver, S., On the quadrature of multivariate highly oscillatory integrals over non-polytope domains, Numer. Math., to appear.

[8] Powell, M.J.D., Approximation Theory and Methods, Cambridge University Press, Cambridge, 1961.

[9] Stein, E., Harmonic Analysis: Real-Variable Methods, Orthogonality, and Oscillatory Integrals, Princeton University Press, Princeton, NJ, 1993. 